\title{
Respiratory Tract Infection Related Healthcare Utilisation in UK Children with Down's Syndrome
}

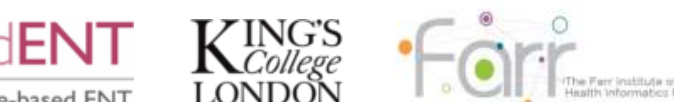

Logan Manikam¹, Meghan Cupp¹, Anne Schilder², Andrew Hayward ${ }^{3}$, Peter Littlejohns ${ }^{4}$, Emma Alexander ${ }^{5}$, Monica Lakhanpaul ${ }^{1}$

1) UCL Institute of Child Health, UK. 2) UCL Ear Institute, UK. 3) UCL Farr Institute of Health Informatics Research, UK. 4) KCL Department of Primary Care and Public Health Sciences, UK. 5) GKT Schools of Medical Education, UK.

Background: Children with respiratory tract infections (RTIs) often present to genera practitioners (GPs), particularly in the winter months when there are higher levels of circulating respiratory tract viruses. For most children, RTls are a mild and short viral illness. However, in certain 'at-risk' children, such as those with Down's Syndrome (DS), RTIs can become more serious and result in hospital admissions (1).

This retrospective cohort study utilised the Clinical Practice Research Datalink (CPRD) and Hospital Episode Statistics (HES) through CALIBER to quantify RTI-related primary and secondary healthcare utilisation and treatment, such as antibiotic prescriptions, in children with DS and controls alongside the risk of hospitalisation from RTIs, time to RTI-related hospitalisation and re-consultation with GPs.

Methods: Individuals were followed up through CALIBER for occurrences of RTIs and antibiotic prescriptions recorded in either the primary or secondary care dataset.

A 28-day window was utilised as the at-risk period for an RTI-related complication or hospitalisation and each consultation for an RTI was followed up for up to 28 days or the first hospitalisation for an RTI within 28 days.

Rates of consultation, hospitalisation and prescription of antibiotics were calculated using Poisson regression. The Wilcoxon test allowed for comparison of hospitalisation length of stay. Analyses were stratified according to year, age, gender, RTI type, season and co-morbidities. Analyses were carried out in STATA version 13.
Results: A total of 992 children with DS aged 0-18 years and 4,874 matched controls were included in this retrospective cohort study using CALIEBR, recording clinical encounters from 1997-2010 (Table 1).

Table 1 - Cohort Demographics

\begin{tabular}{|c|c|c|}
\hline Characteristics & Control $(n=4,874)$ & Children with DS ( $\mathrm{n}=992)$ \\
\hline Male & $2626(53.9 \%)$ & $528(53.2 \%)$ \\
\hline Female & $2248(46.1 \%)$ & $464(46.8 \%)$ \\
\hline Infants (0-1 year) & $1247(25.6 \%)$ & $252(25.4 \%)$ \\
\hline Toddlers (1-5 years) & $1133(23.2 \%)$ & $224(22.6 \%)$ \\
\hline Juniors (5-10 years) & $1044(21.3 \%)$ & $208(21.0 \%)$ \\
\hline Young Persons (10-18 years) & $1454(29.8 \%)$ & $308(29.8 \%)$ \\
\hline Asian or Asian British & $211(2.5 \%)$ & $56(3.3 \%)$ \\
\hline Black or Black British & $189(2.4 \%)$ & $48(2.8 \%)$ \\
\hline Chinese or 'Other' Group & $114(1.35 \%)$ & $30(1.7 \%)$ \\
\hline Mixed Ethnicity & $393(4.7 \%)$ & $72(4.2 \%)$ \\
\hline Unknown Ethnicity & $4005(47.5 \%)$ & $504(29.5 \%)$ \\
\hline White & $3523(41.8 \%)$ & $1001(58.5 \%)$ \\
\hline Asthma & $618(12.7 \%)$ & $136(13.7 \%)$ \\
\hline Congenital heart disease & $48(1.0 \%)$ & $393(39.6 \%)$ \\
\hline Diabetes & $20(0.4 \%)$ & $11(1.1 \%)$ \\
\hline Epilepsy & $34(0.7 \%)$ & $18(1.8 \%)$ \\
\hline Thyroid & $11(0.2 \%)$ & $103(10.4 \%)$ \\
\hline
\end{tabular}

Rates of healthcare utilisation: Children with DS had a rate per person-year of 0.638 (95\% Cl 0.582-0.699) of RTI-related GP consultations, and $0.067 \quad(95 \% \mathrm{Cl}$ 0.0580.077 ) for RTI-related hospitalisations (Figure 1).

RTI-related healthcare utilisation was significantly higher in children with DS compared to controls for both GP consultations (Adjusted RR 1.73; 95\% Cl 1.62-1.84) and hospitalisations (Adjusted RR 5.70; 95\% Cl 4.82-6.71). The differences between children with DS and controls are more pronounced in RTI-related hospitalisations and persisted across all age groups.

Length of hospitalisation: Overall, the length of stay in hospital due to RTIs was longer for children with DS (Mean 5.2; 95\% Cl 5.0-5.4 days per admission) compared to controls (Mean 2.4; 95\% Cl 2.2-2.6) (Figure 2).
Risk of hospitalisation: The risk of an RTIrelated hospitalisation following an RTIrelated GP consultation was higher in children with DS compared to controls (RR $3.15 ; 95 \%$ Cl $2.35-4.24)$. The time to hospitalisation was similar in children with DS (median of 8.0 days; $95 \% \mathrm{Cl} 3.0-19.0$ ) and in controls (median of 8.0 days; $95 \% \mathrm{Cl} 2.0$ 18.0).

Figure 3 - Time to hospitalisation in children with DS and controls following an RTI-related GP

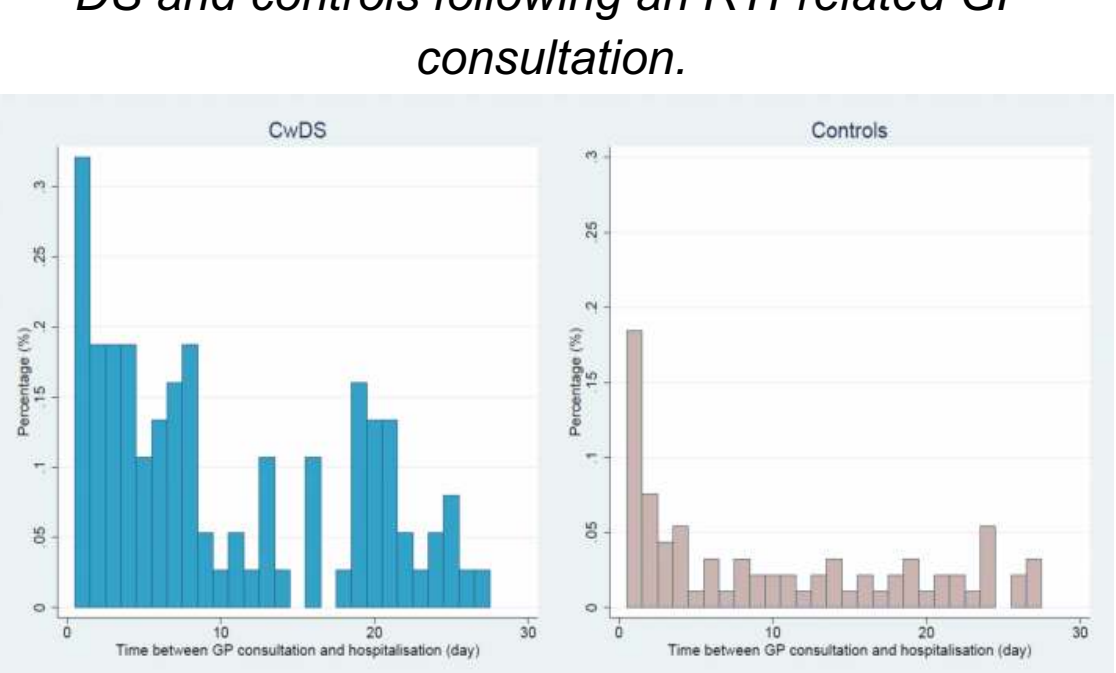

Rates of antibiotic prescribing: Antibiotic prescribing was also consistently higher in children with DS compared to controls (Adjusted RR 2.34; 95\% Cl 2.19-2.5)

Re-consultations: The odds of re-consultation with a GP for an RTI was higher in children with DS (OR 1.69; 95\% Cl 1.57-1.82).

Figure 1 - Annual RTI-related GP consultation and hospitalisation rates in children with $D S$ compared to controls

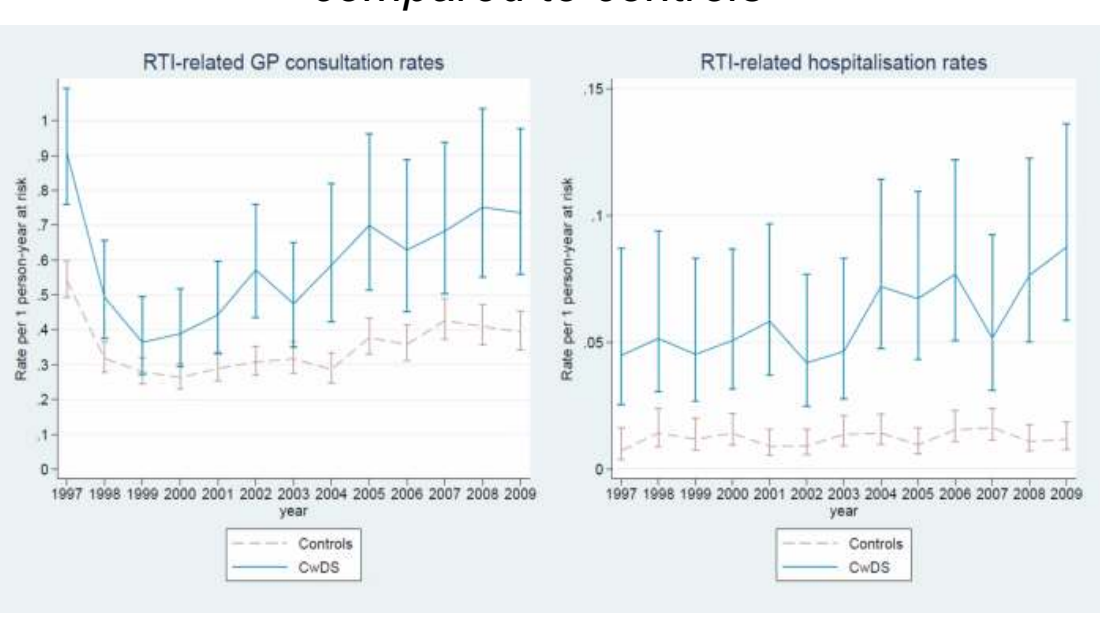

Conclusion: This is the first study of RTIrelated healthcare utilisation in children with DS compared to controls utilising linked primary and secondary care data.

This study demonstrated that children with DS attend GP consultations more often, are hospitalised more often, stay in hospital longer, and are prescribed antibiotics more frequently for RTIs compared to controls. These findings were shown to be individually independent of age, gender, and comorbidities that may influence RTI-related healthcare utilisation, such as CHD and asthma.

These findings are in keeping with previous studies, where children with DS have more hospitalisations and longer hospital stays compared to the general population (2).

Notably, children with DS were three times as likely to be admitted to hospital for an RTI following an RTI-related GP consultation and were prescribed over twice as many antibiotics for RTIs.

Further research should examine prescribing rates for antivirals for influenza in children with DS.

Figure 2 - Distribution of RTI-related hospitalisation length of stay in children with DS

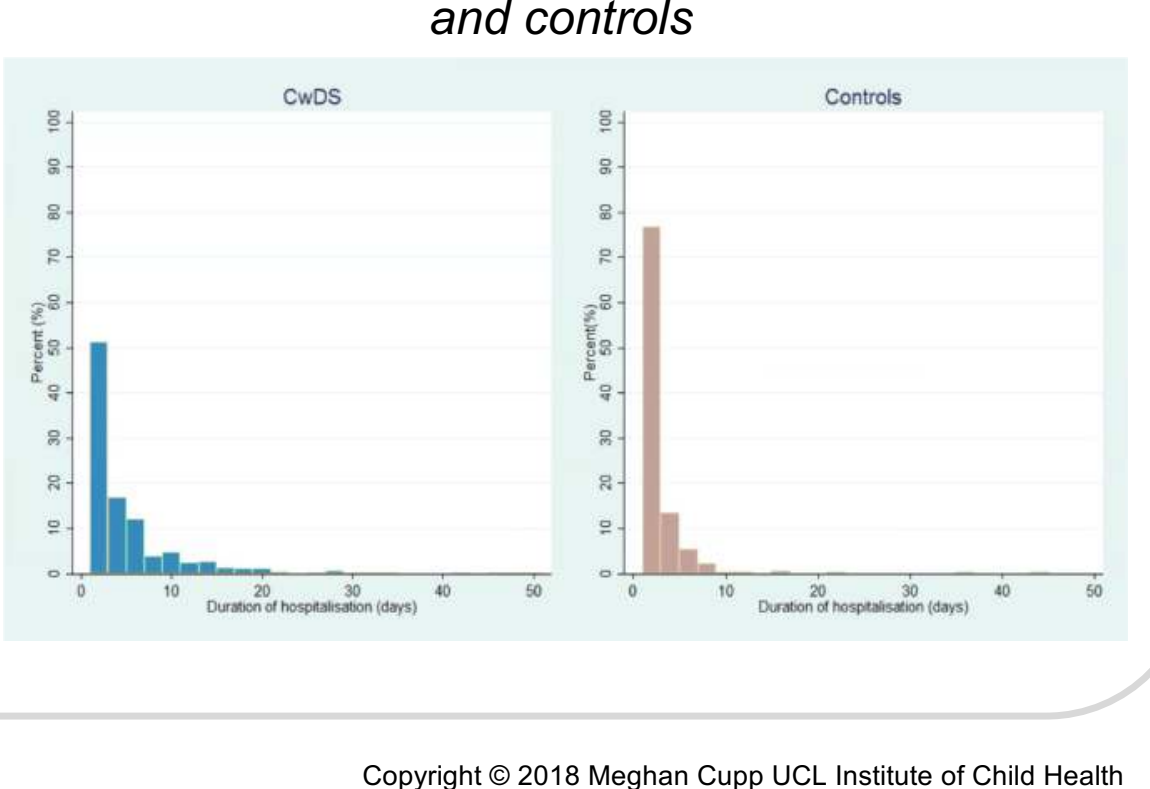

